\title{
Ascendobifemoral Bypass for the Treatment of a Thoracic Endograft Infection
}

\author{
Sebastian Paul Pleger ${ }^{1} \quad$ Nadine Nink$^{1} \quad$ Andreas Böning $^{2} \quad$ Ahmed Koshty $^{1}$
}

\author{
${ }^{1}$ Department of Vascular and Endovascular Surgery, Jung-Stilling \\ Hospital, Siegen, Germany \\ 2 Department of Adult and Pediatric Cardiovascular Surgery and \\ Vascular Surgery, University Hospital of Giessen, Justus Liebig \\ University, Giessen, Germany
}

Thorac Cardiovasc Surg Rep 2017;6:e32-e34.

\begin{abstract}
Address for correspondence Sebastian Paul Pleger, MD, Department of Vascular and Endovascular Surgery, Jung-Stilling Hospital, Siegen, Germany (e-mail: plegersebastian@yahoo.de).
\end{abstract}

\begin{abstract}
Keywords

- endograft infection

- TEVAR

- ascending aorta

- ascendobifemoral bypass

- extra-anatomic

- revascularization

Background Endograft infections (Els) are rare complications after endovascular procedures in the thoracic and abdominal aortas. The challenging treatment encloses antibiotic and surgical therapies.

Case Description A 74-year-old male patient developed an El after an endovascular procedure (thoracic endovascular aortic repair [TEVAR]). Despite a long-term oral antibiotic therapy, the clinical symptoms showed no falling trend. Because of the expanded infection from above the celiac trunk up to the aortic arch, we decided to remove the infected endograft and to implant an extra-anatomic ascendobifemoral bypass.

Conclusion The implantation of an ascendobifemoral bypass was a successful treatment option for Els after TEVAR.
\end{abstract}

\section{Introduction}

The endovascular therapy became the first option for treating patients with abdominal and thoracic aortic aneurysms, aortic dissections (Stanford Type B, BeBakey III), intramural hematoma, and penetrating aortic ulcer (PAU). ${ }^{1,2}$ In comparison to the open surgical procedures, it is associated with a lower risk of intraoperative trauma, less mortality and morbidity, as well as shorter hospital stay. Despite advantages, complications after endovascular aortic repair (EVAR) and thoracic EVAR (TEVAR) require open surgical measures. Especially infections of aortic endografts with high mortality rates are a challenge. ${ }^{3}$ Endograft infections (EIs) might occur in 0.2 to $5 \%$ of the patients. ${ }^{3,4}$ The constitutional symptoms are fever, malaise, thoracic and abdominal pain, weight loss, hematochezia (aortoenteric fistula), and hemoptysis (aortoesophageal or aortobronchial fistula)., ${ }^{5,6}$ Perioperative stent graft seeding, hematogenous seeding, and mechanical erosion of the stent graft are the proposed mechanisms of EI. ${ }^{5}$ Staphylococcus aureus and Streptococcus occur as the

received

June 14, 2017 accepted after revision September 5, 2017
DOI https://doi.org/

$10.1055 / \mathrm{s}-0037-1607272$. ISSN 2194-7635. most frequently isolated bacteria from blood cultures. ${ }^{1,3,5,6}$ The computed tomography angiography (CTA) and fluorodeoxyglucose positron emission tomography are regarded as leading imaging techniques for diagnosis of EI. Despite follow-up protocols after endovascular procedures, there are no screening strategies to detect EI. ${ }^{1}$ The treatment of EI encloses antibiotic therapy and in situ or extra-anatomic reconstructions. Current in situ procedures include aortoaortic, aorto-bi-iliac, or aortobifemoral bypasses and for the extra-anatomic reconstruction, axillofemoral or axillobifemoral bypass. In addition, extra-anatomic procedures such as ascending to descending aortic bypass and ascendoabdominal aortic bypass can be applied. The materials for reconstruction are autologous femoral vein, cryopreserved allografts, rifampicin soaked grafts, and prosthetic grafts. $^{3,4,6-9}$ In this case report, we describe the medical history of a patient with an initial treatment of an EI after TEVAR with an uncommon use of the ascending aorta (AA) as donor artery for the implantation of an ascendobifemoral bypass. (c) 2017 Georg Thieme Verlag KG
Stuttgart · New York

License terms

$\Theta(1) \Theta \Theta$ 


\section{Case Description}

A 74-year-old male patient was referred by the general practitioner to our outpatient department with intermitted fever, progressive back pain, and recently occurring hemoptysis. The laboratory values indicating an inflammation were elevated. One month ago, he was diagnosed with a ruptured PAU and subsequently treated with TEVAR. Despite a longterm oral antibiotic therapy, the clinical symptoms did not ameliorate. We hospitalized the patient and started intravenous antibiotic therapy immediately. Blood cultures revealed the presence of $S$. aureus. The CTA showed gas collections around the aortic stent graft what confirmed the EI ( - Fig. $\mathbf{1}$ ). The infection expanded retrograde right above the celiac trunk up to the aortic arch including the left-sided common carotid artery (CCA). By lacking effect after the antibiotic therapy, we discussed to implant an extra-anatomic ascendobifemoral bypass and performed the procedure after counseling with the patient. We intended to keep both prosthesis limbs retrosternal through the mediastinum and intra-abdominal direct below the abdominal wall. On account of the necessary sternotomy with clamping of the AA, the cooperation with cardiac surgeons was necessary. The whole surgery was divided in two stages. In the first stage, after a minimal sternotomy, the AA was prepared and clamped tangentially without the assistance of the heart lung machine. For the safety of the cerebral and brachial perfusion, a bypass (Dacron graft $14 \times 7 \mathrm{~mm}$, Vascutek) between the AA and the left-sided CCA and subclavian artery was performed (-Fig. 2). Following this procedure, the aortic arch was interrupted distally of the brachiocephalic trunk as a first step of the complete resection of the infected aortic stent graft. Subsequently, the ascendobifemoral bypass was implanted. After the proximal end-to-side anastomosis to the AA (Dacron graft $20 \times 10 \mathrm{~cm}$, Vascutek), both prosthesis limbs were laid retrosternal and intra-abdominal underneath the abdominal wall to the inguinal region. Following this, an anastomosis to the both-sided common femoral arteries was performed (- Fig. 3). In the second stage, following a left-sided thoracotomy, we ligate the thoracic abdominal aorta proximal of the celiac trunk and explanted the infected stent graft entirely (-Fig. 2). During the first postoperative week at the intensive care unit, the renal and

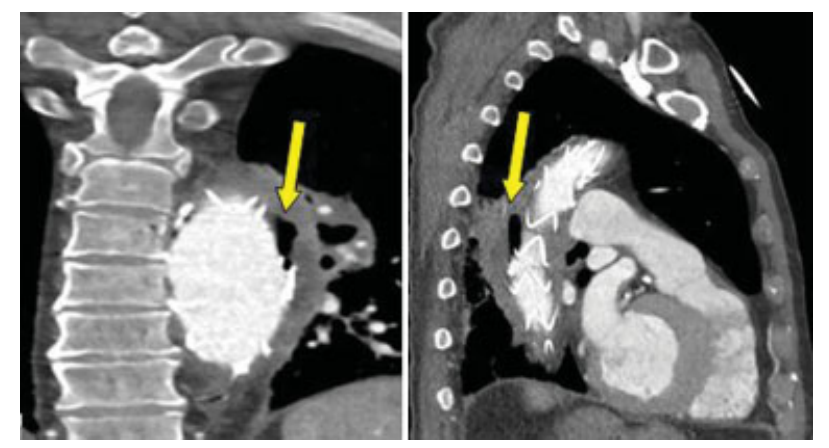

Fig. 1 Computed tomography angiography images with gas collections surrounding the thoracic stent graft (yellow arrows).

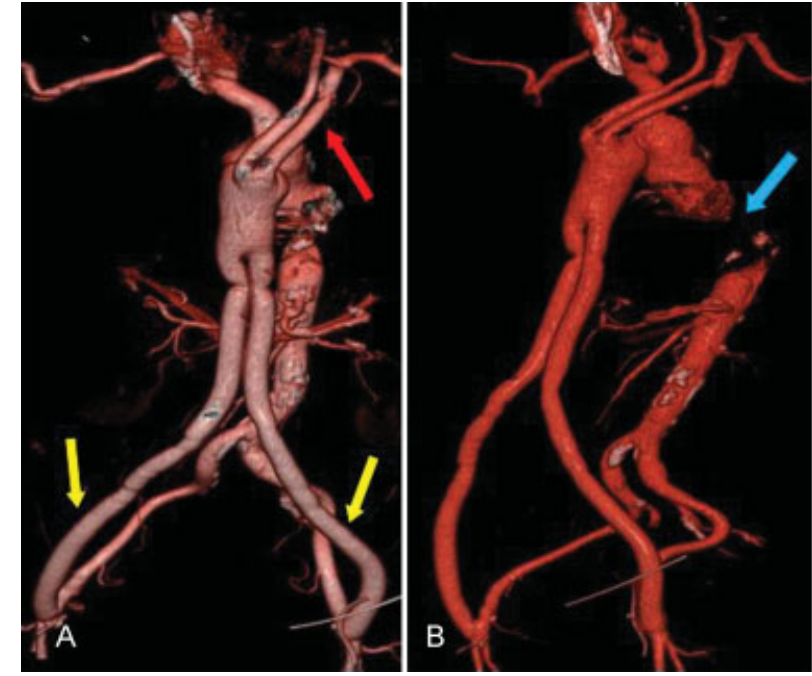

Fig. 2 Three-dimensional computed tomography angiography images of the whole reconstruction with (A) the aortocarotidalsubclavian bypass (red arrow) and prosthesis limbs leading to the femoral arteries (yellow arrows) and (B) the explanted aorta section.

mesenteric system showed no dysfunctions. The inflammation parameters decreased continuously. Neurological symptoms did not appear. For prophylaxis of embolic events, we established a therapy consisting of aspirin and low-molecular-weight heparin. Over the subsequent weeks, the patient developed several comorbidities such as renal insufficiency with the need for dialysis, spondylodiscitis, cholangitis, and respiratory insufficiency with prolonged intubation with resulting pneumonia. Two months after surgery, the patient died due to a sepsis.

\section{Discussion}

According to the international data, $\mathrm{EI}$ is a rare complication after EVAR and/or TEVAR. However, in both cases, treatments remain challenging. The origin of EI is not yet completely cleared. Especially persistent endoleaks are assumed as the most important origin of thrombus seeding, which leads to an increased likelihood of EI. ${ }^{5}$ Animal experiments show that a relevant preventing factor for EI is the formation of an endothelial covering during the neointimal healing process. ${ }^{5}$ The use of antibiotics for avoiding hematogenous bacterial graft seeding usually occurs preoperatively and is continued postoperatively often for a period of 4 or 6 months. As EI mostly become obvious during the first 3 months after surgery, a continuous antibiotic therapy is recommended for 12 months or even lifelong. ${ }^{5,6}$ Patients with good response to antibiotics, a low expansion of the infection, and high perioperative risk can be treated in the long term conservatively. In the presence of extended local infections, inefficient antibiotic respond, and an aortoesophageal fistula, a total endograft excision with surgical reconstruction is recommended. ${ }^{4,6,8}$ In particular, the infection of the thoracic aorta shows a high mortality, which is caused by the complex reconstruction procedures. ${ }^{3}$ Reviewing the literature mortality rates for nonexcisional therapy of infected aortic endografts range from 33 to $70 \%^{3}$ and for 

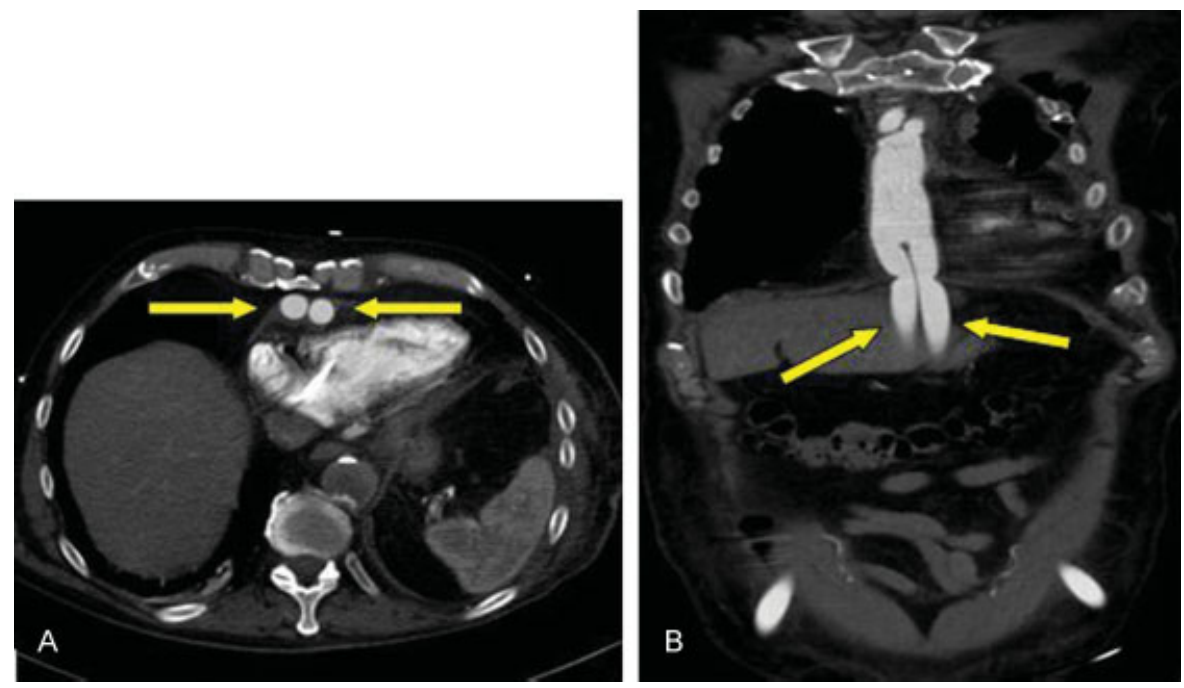

Fig. 3 Computed tomography angiography images with the (A) retrosternal and (B) abdominal.

surgical procedures from 51 to $84 \%$ after 5 years of followup. ${ }^{1,3,4,8}$ In our case, the pronounced cranial and caudal infections of the aorta including the left-sided CCA and subclavian artery as well as the proximity to the celiac trunk lead us to the decision for an extra-anatomic reconstruction. Guided by the widely accepted consensus for treatment with a completely explantation of the infected aortic area, ${ }^{1,6,7}$ we decided for an extra-anatomic reconstruction using the AA as a donor artery for revascularization. With the performance of the ascendobifemoral bypass instead of ascendoabdominal aortic bypass, we intended to avoid, in particular, a manipulation within the infected abdominal aortic area. In addition, we intended to keep a short duration and avoid a two-cavity procedure. Complications such as bypass flow reduction caused by stenosis of the diaphragm section (-Fig. 3) causing legs ischemia or cerebral embolization by manipulating at the aortic arch did not appear. Our case indicated performing the procedure described earlier is safe, and complications such as brain embolization or other ischemic events could be avoided. To our knowledge, the literature shows no similar case for this kind of surgical procedure; wherefore, long-term outcome for the extra-anatomic ascendobifemoral bypass is not known. To prevent EI in the future, there is a need for more studies concerning the exact postoperative application period of antibiotics and the development of screening strategies for early EI detection.

\section{References}

1 Cernohorsky P, Reijnen MM, Tielliu IF, van Sterkenburg SM, van den Dungen JJ, Zeebregts CJ. The relevance of aortic endograft prosthetic infection. J Vasc Surg 2011;54(02):327-333

2 Evangelista A, Czerny M, Nienaber C, et al. Interdisciplinary expert consensus on management of type $B$ intramural haematoma and penetrating aortic ulcer. Eur J Cardiothorac Surg 2015;47(02): 209-217

3 Fatima J, Duncan AA, de Grandis E, et al. Treatment strategies and outcomes in patients with infected aortic endografts. J Vasc Surg 2013;58(02):371-379

4 Smeds MR, Duncan AA, Harlander-Locke MP, et al; Vascular LowFrequency Disease Consortium. Treatment and outcomes of aortic endograft infection. J Vasc Surg 2016;63(02):332-340

5 Heyer KS, Modi P, Morasch MD, et al. Secondary infections of thoracic and abdominal aortic endografts. J Vasc Interv Radiol 2009;20(02):173-179

6 Murphy EH, Szeto WY, Herdrich BJ, et al. The management of endograft infections following endovascular thoracic and abdominal aneurysm repair. J Vasc Surg 2013;58(05):1179-1185

7 Said SM, Burkhart HM, Dearani JA, Connolly HM, Schaff HV. Ascending-to-descending aortic bypass: a simple solution to a complex problem. Ann Thorac Surg 2014;97(06):2041-2047, discussion 2047-2048

8 Oderich GS, Bower TC, Cherry KJ Jr, et al. Evolution from axillofemoral to in situ prosthetic reconstruction for the treatment of aortic graft infections at a single center. J Vasc Surg 2006;43(06): 1166-1174

9 Kumar MV, Choudhary SK, Talwar S, et al. Extraanatomic bypass to supraceliac abdominal aorta for complex thoracic aortic obstruction. Ann Thorac Surg 2016;101(04):1552-1557 